\section{Epidemiology and partner notification \\ P26 THE EFFECTIVENESS OF PARTNER NOTIFICATION IN DIAGNOSING EARLY HIV INFECTION IN PLYMOUTH, UK}

doi:10.1136/sextrans-2012-050601c.26

Z Warwick, ${ }^{*}$ T Wimpenny. Plymouth Hospitals NHS Trust, Plymouth, UK

Background The aim of partner notification (PN) in HIV is to diagnose the undiagnosed and reduce the risk of onward transmission. Plymouth is thought to be a low HIV prevalence area and as such routine testing across medical settings is unlikely to be cost effective or practical. As part of our attempts to target testing, we have concentrated and improved our HIV contact tracing. We describe how the recent infection testing algorithm (RITA) has allowed us to focus PN and identifies individuals early in infection (infectious, high behavioural risk, untested) therefore reducing onward transmission. Aim To describe the number of recent infections diagnosed in Plymouth using PN.

Methods RITA is performed in all newly diagnosed patients. This information is taken into account during PN. A retrospective review of all RITA results done in 2011 and how this has contributed to PN in Plymouth GUM is described.

Results Of the 20 new HIV diagnoses made in Plymouth, 7 (35\%) were incident infections and six were diagnosed as a direct result of PN. Of those with prevalent infection $(n=12), 5$ were diagnosed as a direct result of PN.

Conclusions In low prevalence areas targeted testing will yield the greatest number of positive individuals per test. Along with other targeting strategies, $\mathrm{PN}$ in those newly diagnosed is a way of reaching an at risk group within this setting. It is important to prioritise HIV PN through a dedicated Health Advising team.

\section{P27 AN AUDIT ON THE MANAGEMENT OF PELVIC INFLAMMATORY DISEASE (PID)}

doi:10.1136/sextrans-2012-050601c.27

S Y Chan, ${ }^{*}$ A Hegazi, A Beardall, P Hay. St Georges Hospital, London, UK

Background The 2005 (revised in 2011) UK national guidelines on the management of PID state two auditable outcomes: (1) Proportion of women receiving treatment with a recommended regimentarget $95 \%$. (2) Proportion of named male contacts screened for infection and/or treated-target 0.4 (large urban centres) or 0.6 (other centres) per index case. The guidelines also recommend patients with moderate or severe PID are reviewed after $72 \mathrm{~h}$ and all patients are reviewed after 2-4 weeks.

Aims To audit the management, follow-up and partner notification (PN) of PID in our clinic.

Methods Retrospective notes review of PID cases presenting between December 2009 and July 2011. Data were collected on drug treatment, follow-up and PN. Cases with a sexually transmitted infection (STI) were compared to cases with no STI identified.

Results Of 130 PID cases identified, 58 had an STI diagnosed of whom seven had Neisseria gonorrhoeae (GC) and 57 had Chlamydia trachomatis (CT). Six cases had both GC and CT. One patient had no documentation of drugs prescribed and one received only Doxycycline (see abstract P27 table 1).

Conclusion BASHH guidelines recommend GC cover for all PID cases but the proportion receiving this was $72.9 \%$. All cases diagnosed with GC received GC cover. PN rates did not reach national recommendations and this may be related to diagnostic uncertainty especially when no STI is apparent. PN was better in cases with a STI where health advisers were involved than in cases without an STI, seen by doctors. No patients were advised to return for followup within 3 days but the majority returned within 2-4 weeks.
Those with an STI diagnosed were significantly more likely to attend follow-up $\mathrm{p}<0.0001$.

Abstract P27 Table 1 Management of patients diagnosed with PID

\begin{tabular}{lcc}
\hline & $\begin{array}{l}\text { STI identified } \\
(\mathbf{n}=\mathbf{5 8})\end{array}$ & $\begin{array}{l}\text { No STI identified } \\
(\mathbf{n = 7 2})\end{array}$ \\
\hline Partner notification (PN) & & $19(26.4 \%)$ \\
$\quad$ Partner notified & $21(36.2 \%)$ & $5(6.9 \%)$ \\
Partner notified and treated & $20(34.5 \%)$ & 0 \\
PN recommended & $6(10.3 \%)$ & $53(73.6 \%)$ \\
No documentation of PN & $31(53.5 \%)$ & $59(81.9 \%)$ \\
Treatment prescribed & & $7(9.7 \%)$ \\
Doxycycline and metronidazole & $46(79.3 \%)$ & $5(7.0 \%)$ \\
Macrolide and metronidazole & $6(10.4 \%)$ & $44(61.1 \%)$ \\
Ofloxacin and metronidazole (GC cover) & $5(8.6 \%)$ & 0 \\
Cephalosporin prescribed (GC cover) & $40(69.0 \%)$ & $69(95.8 \%)$ \\
Follow-up & & $29(40.3 \%)$ \\
Follow-up advised in 3 days & 0 & $47(81 \%)$ \\
Follow-up advised in 2-4 weeks & $47(81 \%)$ & \\
Follow-up attended &
\end{tabular}

P28

A RE-AUDIT OF PARTNER NOTIFICATION IN HIV POSITIVE INDIVIDUALS IN PLYMOUTH

doi:10.1136/sextrans-2012-050601c.28

T Wimpenny, ${ }^{*}$ Z Warwick. Plymouth Hospitals NHS Trust, Plymouth, UK

Aims Following an audit in 2010 we wanted to establish whether changes in our approach to $\mathrm{PN}$ in newly diagnosed HIV patients or patients seen for the first time had improved our HIV PN outcomes and documentation.

Methods A retrospective case note review was carried out in patients attending the clinic for the first time in 2011. We looked at whether patients had a partner history completed for the look back period, how many partners were contactable and whether the outcome of the $\mathrm{PN}$ was recorded. These results were compared with a similar audit carried out in 2010 .

Results 31 patients were included in the re-audit, 12 transfers into Plymouth GUM and 19 newly diagnosed HIV positive patients. $77 \%$ were male and $23 \%$ were MSM. $100 \%$ of patients had a contact tracing proforma in their notes and 97\% (30/31) had been completed. $36(40 \%)$ partners were untraceable. Of those which were contactable13 were current partners and HIV +; 16 ex-partners who were HIV+; 15 ex-partners verified as HIV-; 7 current partners tested HIV-; two partners remain untested. There were 13 patients who transferred into the department in 2010, three of whom had $\mathrm{PN}$ performed since joining our clinic. There were 13 new diagnoses, 10 of whom had documented CT.

Conclusion PN can be significantly improved using audit in this way. A dedicated Health Advising team is essential where targeted testing for HIV is likely to be the most efficient way of detecting undiagnosed HIV.

\section{P29 AN AUDIT OF HIV PARTNER NOTIFICATION PRACTICES IN THE NORTH EAST}

doi:10.1136/sextrans-2012-050601c.29

${ }^{1} \mathrm{G}$ Dolan, ${ }^{*}{ }^{1} \mathrm{~K}$ Foster, ${ }^{2} \mathrm{~K}$ Kain. ${ }^{1}$ Health Protection Agency-North East; ${ }^{2}$ Newcastle Hospitals Community Health, Newcastle upon Tyne, UK

Background Late diagnosis of HIV not only carries a risk of poorer outcomes for the individual, but of ongoing transmission of 